\title{
Transient dynamics in dense colloidal suspensions under shear: shear rate dependence
}

\author{
M Laurati ${ }^{1}$, K J Mutch ${ }^{1}$, N Koumakis ${ }^{2}$, J Zausch $^{3}$, C P Amann ${ }^{5}$, \\ A B Schofield ${ }^{6}$, G Petekidis ${ }^{2}$, J F Brady ${ }^{7}$, J Horbach $^{4}$, M Fuchs ${ }^{5}$ and \\ S U Egelhaaf ${ }^{1}$ \\ 1 Condensed Matter Physics Laboratory, IPkM, Heinrich-Heine University, 40225 Düsseldorf, Germany \\ ${ }^{2}$ FORTH/IESL and Department of Materials Science and Technology, University of Crete, 71110 , \\ Heraklion, Greece \\ ${ }^{3}$ Fraunhofer ITWM, Fraunhofer-Platz 1, 67663 Kaiserslautern, Germany \\ ${ }^{4}$ Theoretical Physics II: Soft Matter, Heinrich-Heine University, 40225 Düsseldorf, Germany \\ ${ }^{5}$ Fachbereich Physik, University of Konstanz, D-78457 Konstanz, Germany \\ ${ }^{6}$ SUPA, School of Physics and Astronomy, The University of Edinburgh, Edinburgh EH9 3JZ, UK \\ ${ }^{7}$ Division of Chemistry and Chemical Engineering, California Institute of Technology, Pasadena, \\ CA 91125 , USA \\ E-mail: marco.laurati@uni-duesseldorf.de
}

\begin{abstract}
A combination of confocal microscopy and rheology experiments, Brownian dynamics (BD) and molecular dynamics (MD) simulations and mode coupling theory (MCT) have been applied in order to investigate the effect of shear rate on the transient dynamics and stress-strain relations in supercooled and glassy systems under shear. Immediately after shear is switched on, the microscopic dynamics display super-diffusion and the macroscopic rheology a stress overshoot, which become more pronounced with increasing shear rate. MCT relates both to negative sections of the generalized shear modulus, which grow with increasing shear rate. When the inverse shear rate becomes much smaller than the structural relaxation time of the quiescent system, relaxation through Brownian motion becomes less important. In this regime, larger stresses are accumulated before the system yields and the transition from localization to flow occurs earlier and more abruptly.
\end{abstract}

\section{Introduction}

The glass transition of colloidal hard spheres has attracted considerable scientific interest for many ycars [1-3]. The behaviour of hard spheres is controlled by the volume fraction $\phi$, i.e. the fraction of the sample volume occupied by particles. For $\phi \gtrsim 0.58$, slightly polydisperse samples do not show crystallization, which is the thermodynamically preferred state, but remain in an amorphous, glassy non-equilibrium state. The glass transition seems to coincide with the onset of structural arrest, suppression of long-distance diffusion and partial freezing of density fluctuations [4-6]. This can be qualitatively explained on the basis of the cage effect: as $\phi$ increases, particles are increasingly hindered in their motions, i.e. 'caged', by their nearest neighbours until they become localized and dynamical arrest takes place. This can be theoretically described by mode coupling theory (MCT) [7, 8]. Ageing and hopping processes are believed to allow particles to escape from their cages and can lead to long-time diffusion and flow $[3,9]$.

The glass transition is reflected in the linear regime of the rheological response: dynamical arrest leads to 
a solid-like response [10]. Whilst the elastic response dominates, dissipative contributions are nevertheless present due to activated processes leading to long-time relaxation. The linear viscoelastic properties can be described by using MCT-based theories [10, 11].

Upon exceeding the yield stress or strain, the glass begins to plastically deform $[12,13]$ and the relation between the applied field and the system's response becomes non-linear. The steady non-linear response of concentrated dispersions and glasses to shear has been investigated in experiments $[11-16]$, simulations $[17,18]$ and theory $[9,19,22-25]$. In the glass, these studies indicate that this state is characterized by a finite value of the yield stress. Under application of a constant shear rate, glasses start to flow; simulations [18] and experiments [24-26] show that the non-alfine motions of the particles, initially arrested, become diffusive once shear is applied for some time. The long-time diffusion coefficient is controlled by the shear rate $\dot{\gamma}$, the only control parameter. Only some time after the commencement of shear is the steady state of flow established. A transient regime occurs during which the stress-strain relation displays an overshoot [26-29], which has also been observed in other soft materials [29-36]. At comparable strains, a pronounced structural anisotropy of the cage develops [26, 37, 38] that does not fully relax even in the steady state of shear. Furthermore, in a similar range of strains, the transient dynamics show super-diffusive motion [26, 27, 39]. Using MCT, these phenomena have been related to negative sections of the generalized shear modulus [27]. However, the dependence of the transient rheological response, structure and dynamics on the control parameters, in particular the shear rate $\dot{\gamma}$, i.e. the scaled Peclet number $P e=\dot{\gamma} t_{\mathrm{B}}$, where $t_{\mathrm{B}}$ is the Brownian relaxation time, or the Weissenberg number $P e^{w}=\dot{\gamma} \tau_{R}$, where $\tau_{R}$ is the structural relaxation time, have not been investigated so far.

Here we investigate the shear rate dependence of the microscopic particle dynamics and macroscopic rheology of concentrated colloidal suspensions under steady shear and while steady shear develops. We present results obtained using different approaches: experiments, Brownian dynamics (BD) simulations of hard spheres, molecular dynamics (MD) simulations of a binary Yukawa mixture, and MCT calculations. This also allows us to investigate the influence of interactions on the transient phenomena. We limit our study to shear rates where homogeneous flow is observed, i.e. we avoid combinations of volume fractions and shear rates which produce non-homogeneous velocity profiles with shear banding $[40,41]$.

\section{Materials and methods}

\subsection{Samples}

Polymethylmethacrylate (PMMA) colloids sterically stabilized with a layer of polyhydroxystearic acid (PHS) and fluorescently labelled with nitrobenzoxadiazole (NBD) were dispersed in a mixture of cycloheptyl bromide and cis-decalin that closely matches the density and refractive index of the colloids. In this solvent mixture, the particles acquire a small charge which is screened by adding $4 \mathrm{mM}$ tetrabutylammoniumchloride [42]. The system displays almost hard-sphere behaviour, with the volume fraction $\phi=(4 \pi / 3) n R^{3}$ being the only thermodynamic control parameter, with $n$ the particle number density and $R$ the particle radius.

The radius $R=770 \pm 6 \mathrm{~nm}$ was determined by means of static light scattering on a very dilute colloidal suspension $\left(\phi \simeq 10^{-4}\right)$. This value is similar to that estimated from the position of the first peak of the radial distribution function obtained by confocal microscopy [43], which was found to be $R=780 \mathrm{~nm}$. The very dilute sample was also used for dynamic light scattering measurements; the radius extracted from the diffusion coefficient, $D_{0}$, was found to be in agreement with the other values. From the angular dependence of the diffusion coefficient, a relative polydispersity in size of 0.062 was determined [44]. Samples for confocal microscopy and rheology were prepared from a random-close-packed (RCP) stock solution which was obtained by sedimenting a dilute colloidal suspension in a centrifuge. Given a polydispersity of 0.062 , the sediment is expected to have a volume fraction $\phi \approx 0.66$ [45]. Subsequently, the sample was diluted and its volume fraction determined as follows. The imaged volume was partitioned into Voronoï cells and the mean size of the Voronoï volume per particle determined. The ratio of the particle volume to the mean Voronoî volume provides an estimate of the volume fraction of the sample, $\phi=0.56 \pm 0.01$ [46]. In order to improve the signal-to-noise ratio in the rheology measurements, similar, but smaller and not fluorescently labelled particles with radius $R=267 \pm 3 \mathrm{~nm}$ and a polydispersity of about 0.06 (from dynamic light scattering), dispersed in cis-trans-decalin, were used. Samples of these smaller particles were prepared from a stock solution with the volume fraction determined in the fluid-solid coexistence region [47].

\subsection{Confocal microscopy under shear}

Shear was applied to suspensions of the large spheres by means of two parallel plates using a home-built shear cell (sketched in figure 1) $[27,48]$. In order to prevent wall slip, the plates, two glass coverslips, were coated with a layer of very polydisperse PMMA particles [24]. Before each experiment was conducted, a rejuvenation protocol was performed in order to reduce history effects on the sample. This consisted of applying typically 10 large amplitude oscillations (at a strain in excess of 100\%) at a frequency below $0.1 \mathrm{~Hz}$, and subsequently waiting $600 \mathrm{~s}$ before starting the experiment.

Confocal microscopy experiments were performed using a VT-Eye confocal microscope (Visitech International) mounted on a Nikon TE2000-U inverted microscope and using a Nikon Plan Apo VC $100 \times$ oil immersion objective. Two-dimensional images of the samples were recorded at a depth of $30 \mu \mathrm{m}$ inside the sample in order to probe bulk behaviour and to retain good quality images. The image size was chosen to be 512 pixels $\times 512$ pixels, corresponding to an area of $57 \times 57 \mu^{2}$. A series of images was acquired at 


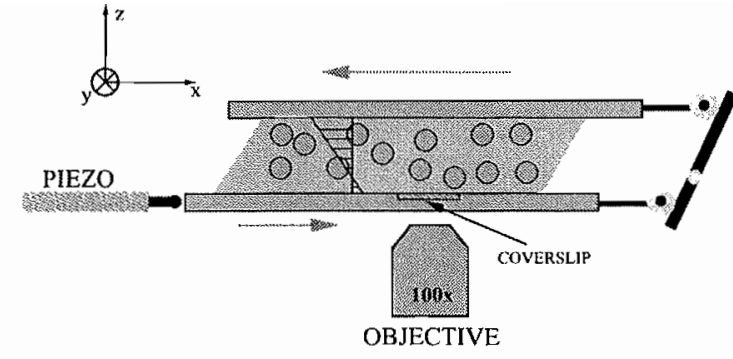

Figure 1. Schematic representation of the parallel plate shear cell used in combination with confocal microscopy. A piezo-actuator drives the bottom plate in the positive $x$ direction and a rotating lever transfers the movement to the upper plate. The axis of rotation of the lever can be translated vertically, thereby changing the amplification of the movement of the bottom plate. The sample is contained between microscope coverslips glued to the plates. The velocity $(x)$, vorticity $(y)$ and gradient $(z)$ directions are indicated. The velocity profile, represented by the black lines in the sample, contains a zero-velocity plane due to the movement of the plates in opposite directions.

a fast sampling rate (compared to the shear rate) during the application of shear. Additional experimental details can be found in previous work [27].

Particle coordinates and trajectories were extracted from the confocal images using standard routines [49]. The shear rates applied here were sufficiently small that particles did not move very far between two consecutive frames, even in the direction of shear $(x)$. It was thus not necessary to remove affine motions before tracking [25]. Particle trajectories were used to calculate mean squared displacements (MSD) $\left\langle\Delta y^{2}\left(t, t_{\mathrm{w}}\right)\right\rangle$ as a function of time $t$ in the vorticity direction $(y)$ :

$$
\left\langle\Delta y^{2}\left(t, t_{\mathrm{W}}\right)\right\rangle=\left\langle\frac{1}{N} \sum_{i=1}^{N}\left(y_{i}\left(t+t_{\mathrm{w}}\right)-y_{i}\left(t_{\mathrm{w}}\right)\right)^{2}\right\rangle
$$

where $t_{\mathrm{w}}$ is the waiting time, $N$ is the number of particles found in an image, typically about 1200 , and \langle\rangle indicates the average over independent experiments. In order to study the dependence on waiting time $t_{\mathrm{w}}$, only trajectories starting at a time $t_{\mathrm{w}}$ after the commencement of shear are included. This significantly reduces the statistics compared to measurements at rest, where an average over $t_{\mathrm{w}}$ can be performed and hence trajectories starting at different times are taken into account. In order to improve the statistics, MSDs extracted from typically ten experiments were averaged after checking the reproducibility of the measurements.

\subsection{Rheology}

Rheological measurements for samples of small spheres were performed on a stress controlled Anton Paar MCR-501 rheometer at $T=20^{\circ} \mathrm{C}$. A cone and plate geometry with a diameter of $50 \mathrm{~mm}$ and an angle of $0.0175 \mathrm{rad}$ was used in order to accommodate the low stress of the sample. The cone truncation was $0.102 \mathrm{~mm}$. Measurements of samples of large spheres were performed using a TA Instruments
ARES-G2 strain controlled rheometer at $T=20^{\circ} \mathrm{C}$ and a cone and plate geometry. A cone of diameter $50 \mathrm{~mm}$ and angle 0.04 rad was used with a truncation gap of $0.048 \mathrm{~mm}$. To reduce statistical noise, 30 measurements for each shear rate were performed, which is at the limit of sample stability. A rejuvenation protocol similar to that used for confocal microscopy experiments was also applied.

\subsection{Simulation models}

In the $\mathrm{BD}$ simulations for the hard-sphere system, hydrodynamic interactions (HI) were ignored, allowing the simulation of highly concentrated colloidal suspensions and larger particle systems, in comparison to the more accurate but computationally more demanding Stokesian dynamics [50] where HI are included. The particle motion in colloidal suspensions is described by the $N$-body Langevin equation:

$$
\mathbf{m}(\mathrm{d} \mathbf{U} / \mathrm{d} t)=\mathbf{F}^{\mathrm{H}}+\mathbf{F}^{\mathrm{B}}+\mathbf{F}^{\mathrm{P}},
$$

where $\mathbf{m}$ is the generalized mass/moment of inertia tensor, $\mathbf{U}$ is the particle translational/rotational velocity vector, $\mathbf{F}^{\mathrm{H}}$ is the hydrodynamic force vector, $\mathbf{F}^{\mathrm{B}}$ is the stochastic force vector that gives rise to Brownian motion, and $\mathbf{F}^{\mathrm{P}}$ is the deterministic non-hydrodynamic force vector. For $N$ rigid particles of radius $R$ and density $\rho$ in a medium of viscosity $\eta$, we study states at a shear rate $\dot{\gamma}$ where the inertial forces are negligible compared to viscous forces, i.e. at small Reynolds number, $R e=\rho R^{2} \dot{\gamma} / \eta \ll 1$, where the above equation reduces to $\mathbf{F}^{\mathrm{H}}+\mathbf{F}^{\mathrm{B}}+\mathbf{F}^{\mathrm{P}}=0$. In $\mathrm{BD}$ (ignoring HI between particles), the hydrodynamic force reduces to Stokes drag: $\mathbf{F}^{\mathrm{H}}=-6 \pi \eta R \mathbf{U}$. For a simple hard-sphere system, the non-hydrodynamic force vector becomes equal to the hard-sphere interaction forces occurring at contact.

The hard-sphere interactions are based on the 'potentialfree' algorithm [51] in which the overlap between pairs of particles is avoided by moving the particles with equal force along the line of centres, back to contact. In order to calculate the stress, the algorithm directly calculates the pairwise interparticle forces that would have resulted in the hard-sphere displacements during the course of a time step [52]. Therefore we may write $\mathbf{F}^{\mathrm{P}}=6 \pi \eta R\left(\Delta x^{\mathrm{HS}} / \Delta t\right)$, the average Stokes drag on the particle during the course of the hard-sphere displacement, which is then used to calculate the rheologically relevant contribution to the stress, $\sigma_{x y}=-N\left\langle\mathbf{x F}^{\mathrm{P}}\right\rangle[51]$. Details of the specific BD simulation method used here can be found elsewhere [52]. At rest and for relatively low $P e$, Brownian dynamics simulations qualitatively capture experimental stresses and displacements, even though $\mathrm{HI}$ are ignored $[50,53]$, but stresses may not be quantitatively correct.

The main details of the BD simulations are as follows: a volume fraction $\varphi=0.56$, with $N=5405$ particles at rates of $P e^{\mathrm{w}}=4.9,49$ and $490\left(P e=0.1,1\right.$ and 10, with $\tau_{\mathrm{R}}=$ $44.4 t_{\mathrm{B}}$ ), was examined using multiple runs (eight runs) in order to average initial configurations. Data for accumulated strains larger than 1 were taken from single runs. To avoid ordering during shear, polydispersity was added, which is represented by a discrete Gaussian distribution of radii with 
80 components and a root mean squared deviation of $10 \%$. The effect of the polydispersity on stresses and displacements was found to be minimal in comparison to that for the monodisperse system.

The MD simulations were performed for a binary $A B$ mixture, modelled by the Yukawa potential,

$$
u_{\alpha \beta}=\epsilon_{\alpha \beta}^{\mathrm{MD}} d_{\alpha \beta} \frac{\mathrm{e}^{\left(-\kappa_{\alpha \beta}\left(r-d_{\alpha \beta}\right)\right)}}{r}
$$

with $\alpha, \beta=\mathrm{A}, \mathrm{B}$. The functions $u_{\alpha \beta}$ are truncated at a cut-off distance $r_{\mathrm{c}}^{\alpha \beta}$, defined by $u_{\alpha \beta}\left(r_{\mathrm{c}}^{\alpha \beta}\right)=10^{-7} \epsilon_{\mathrm{AA}}^{\mathrm{MD}}$. The 'particle diameters' were set to $d \equiv d_{\mathrm{AA}}=1.0, d_{\mathrm{BB}}=1.2 d$ and $d_{\mathrm{AB}}=$ $1.1 d$, the cnergy parameters to $\epsilon^{\mathrm{MD}} \equiv \epsilon_{\mathrm{AA}}^{\mathrm{MD}}=1.0, \epsilon_{\mathrm{BB}}^{\mathrm{MD}}=$ $2.0 \epsilon^{\mathrm{MD}}, \epsilon_{\mathrm{AB}}^{\mathrm{MD}}=1.4 \epsilon^{\mathrm{MD}}$, and the screening parameters to $\kappa_{\mathrm{AA}}=\kappa_{\mathrm{BB}}=\kappa_{\mathrm{AB}}=6 / d$. The masses of the particles were set to unity, i.e. $m=m_{\mathrm{A}}=m_{\mathrm{B}}=1.0 . k_{\mathrm{B}}$ is set to unity. The choice of these parameters ensures that, at the density $\varrho=0.675 m_{\mathrm{A}} / d_{\mathrm{AA}}^{3}$ used in this work, no problems with crystallization or phase separation occur, at least in the temperature range under consideration.

The simulations were performed for a 50:50 mixture of $N=2 N_{\mathrm{A}}=2 N_{\mathrm{B}}=1600$ particles, placed in a cubic simulation box of linear size $L=13.3 d$. Systems were sheared via Lees-Edwards boundary conditions. To keep temperature constant, the system was coupled to a dissipative particle dynamics (DPD) thermostat. Further details on the simulation and the thermostat can be found in [27, 38]. Note that for our Yukawa model at the number density considered, the critical temperature of mode coupling theory is approximately $T_{\mathrm{c}}=0.14$. The shear rates used in the simulations were $6 \times 10^{-5}, 3 \times 10^{-4}, 6 \times 10^{-4}$ and $3 \times 10^{-3}$, corresponding to $P e^{\mathrm{w}}=5.8,29,58$ and 288 , with $\tau_{\mathrm{R}}=9.7 \times 10^{-4}$.

\subsection{Mode coupling theory}

MCT provides, via integration through transients (ITT), a closed set of equations of motion (EOM) for calculating the transient dynamics of dispersions under shear [21, 54-57]. Here, a schematic model which neglects the spatial correlations, but keeps the form and asymptotic behaviour of the full model near the glass transition, is used to describe the mean squared displacements (MSD) as a function of time. The basic version of the model is able to describe linear response moduli, flow curves and stress-strain curves. It has been discussed extensively elsewhere $[11,58,59]$ and is hence only briefly summarized here. The EOM of a density correlator $\Phi(t)$, which encodes structural rearrangements, reads [60]

$$
\begin{aligned}
& \partial_{t} \Phi(t)+\Gamma\left\{\Phi(t)+\int_{0}^{t} \mathrm{~d} t^{\prime} m\left(t-t^{\prime}\right) \partial_{t}^{\prime} \Phi\left(t^{\prime}\right)\right\}=0, \quad \text { with (4) } \\
& m(t)=\frac{v_{1} \Phi(t)+v_{2} \Phi^{2}(t)}{1+\left(\dot{\gamma} t / \gamma_{\mathrm{c}}\right)^{2}}
\end{aligned}
$$

Short-time Brownian motion initiates $\Phi(t)=1-\Gamma t$. The exponential decay rate $\Gamma$ describes the microscopic dynamics on short timescales and depends on structural and hydrodynamic correlations. The memory kernel $m(t)$ captures the dynamic arrest of slow density fluctuations when approaching the volume fraction of the glass transition from below and prevents $\Phi(t)$ from decaying to zcro in the glassy phase (ideal glass). The memory kernel of the frequently used $F_{12}$ model is also used here $[8,61]$. In the denominator of $m(t)$, an additional strain term $\dot{\gamma} t / \gamma_{c}$ with constant shear rate $\dot{\gamma}$ accounts for the shear induced decay of the transient correlator to zero, which captures shear melting. This shear rate generalization is called the $F_{12}^{(\dot{\gamma})}$ mode] $[56,60]$. As a model parameter, $\gamma_{c}$ determines the influence of shear on the memory kernel or, more precisely, the strain scale for shear-driven correlator decay. A parameter set $v_{1}=v_{1}^{\mathrm{c}}+\varepsilon \lambda /(1-\lambda)$, with $v_{1}^{\mathrm{c}}=(2 \lambda-1) / \lambda^{2}, v_{2}=v_{2}^{\mathrm{c}}=$ $1 / \lambda^{2}$, and $1 / 2 \leqslant \lambda \leqslant 1$, describes a bifurcation scenario of the non-ergodicity parameter $f(\varepsilon) \equiv \Phi(t \rightarrow \infty, \varepsilon)$, which is $f(\varepsilon<0)=0$ and $f(\varepsilon>0)>f(0)>0$, with $\varepsilon=\left(\phi-\phi_{\mathrm{g}}\right) / \phi_{\mathrm{g}}$ the separation parameter denoting the relative distance from the glass transition. A value of $\lambda=1 / \sqrt{2}$, i.e. $f^{\mathrm{c}} \equiv f(0)=$ $(1-\lambda) \simeq 0.293$, is often chosen, which we also adopt [8]. These parameters have already been used previously to describe stationary, start-up and large amplitude oscillatory stress measurements $[11,57-59]$.

To describe the shear stress $\sigma(t)$ (with orientational dependences neglected), the numerically determined correlator $\Phi(t)$ is used in our non-linear constitutive equation

$$
\begin{aligned}
\sigma(t, \dot{\gamma}) & =\int_{-\infty}^{t} \mathrm{~d} t^{\prime} g\left(t-t^{\prime}, \dot{\gamma}\right) \dot{\gamma}\left(t^{\prime}\right) \\
& =\dot{\gamma} \int_{0}^{t} \mathrm{~d} s g(s, \dot{\gamma}), \quad \text { with } \\
g(t, \dot{\gamma}) & \equiv v_{\sigma}(t \dot{\gamma}) \Phi^{2}(t, \dot{\gamma})+\eta_{\infty} \delta\left(t-0^{+}\right),
\end{aligned}
$$

where constant shear starts at $t=0$. The generalized shear modulus $g(t, \dot{\gamma})$ is a stress auto-correlation function, which reflects the MCT approximation that stress fluctuations decay due to structural rearrangements (captured in $\Phi(t)$ ) and includes the strain dependence of an effective elastic coefficient (namely $v_{\sigma}(t \dot{\gamma})$ ). It characterizes the magnitude of stress fluctuations and decorrelates with strain due to the affine distortions under flow $[27,59]$. The short-time high-frequency viscosity $\eta_{\infty}$ accounts for viscous processes that require no structural relaxation, e.g. the viscosity of the solvent or hydrodynamic interactions.

In order to observe a stress overshoot, the generalized shear modulus $g(t, \dot{\gamma})$ must cross zero. That this is possible within MCT has been observed [60] and discussed in detail $[27,59]$. In the $F_{12}^{(j)}$ model, a simple strain-dependent form of $v_{\sigma}(t, \dot{\gamma})=v_{\sigma}(\gamma=t \dot{\gamma})$ from equation (7) captures this as

$$
v_{\sigma}(t \dot{\gamma})=v_{\sigma}^{*}\left(1-\left(\frac{\dot{\gamma} t}{\gamma^{*}}\right)^{4}\right) \mathrm{e}^{-\left(\frac{\dot{y} t}{\gamma^{* *}}\right)^{4}} .
$$

The strain parameter $\gamma^{*}$ marks the peak of the stress overshoot and is directly obtained from the experimental stress-strain curve, whilst the strain parameter $\gamma^{* *}$ indicates the strain at which the stress approaches its steady linit corresponding to the flow-curve value.

The MSD of a tracer particle under shear was obtained within microscopic MCT in two dimensions [40]. While 
gencral solutions in three dimensions have previously not been possible, a quite involved model based on a generalized Stokes-Einstein relation provides isotropically averaged spatial information [27]. Hcre the model is simplificd even further. The stress overshoot and super-diffusive particle motion are directly connected within the $F_{\mathrm{I} 2}^{(\dot{\gamma})}$ model. It is based on the generalized Stokes-Einstein relation (GSE) and a link between coherent and incoherent memory functions that would arise in microscopic MCT has been formulated. Microscopic MCT gives for the MSD of a Brownian particle $[8,39,62,63]$

$$
\left\langle y^{2}(t)\right\rangle+D_{0} \int_{0}^{t} \mathrm{~d} t^{\prime} m^{(0)}\left(t-t^{\prime}\right)\left\langle y^{2}\left(t^{\prime}\right)\right\rangle=2 D_{0} t,
$$

with $D_{0}$ the short-time diffusion coefficient describing the local motion of the particle. Introducing just one fitting parameter, $\alpha$, we approximate

$$
m^{(0)}(t, \dot{\gamma})=\frac{6 \pi R \alpha}{k_{\mathrm{B}} T} g(t, \dot{\gamma})
$$

This leads to the familiar approximation for the long-time diffusion coefficient, $D_{\mathrm{L}}^{\mathrm{EQ}} \approx k_{\mathrm{B}} T /(6 \pi R \alpha \eta)$, which connects the diffusion coefficient, $D_{\mathrm{L}}^{\mathrm{EQ}}$, with the long-time shear viscosity, $\eta=\sigma(t \rightarrow \infty) / \dot{\gamma}$ (equation (6)) [10]. Equation (9) is derived within quiescent MCT [8], but holds in specific directions under flow [40]. In contrast, the schematic model does not discriminate between different directions and thus can only be used for the directions perpendicular to the flow. The effect of $\eta_{\infty}$, equation (7), is that of a rescaling of $D_{0}$ through division by $1+D_{0} \eta_{\infty}$, which we consider to be outside the range of applicability of the model; hence $\eta_{\infty}=0$ is chosen for the calculation of the MSDs in the following.

At short times, MD simulations yield ballistic motion, in contrast to the diffusive motion in equation (9). To allow for a better comparison with the MD data, we change $(1 / \Gamma) \partial_{t} \Phi(t)$ to $\left(1 / \Omega^{2}\right) \partial_{t}^{2} \Phi(t)$ in equation (4) and $\left\langle y^{2}(t)\right\rangle$ to $\partial_{t}\left\langle y^{2}(t)\right\rangle$ in equation (9). These two equations lead to short-time ballistic motion, whilst the long-time behaviour is not changed. Evidently these changes are not needed when comparing theory with results of experiments and BD simulations.

\section{Results and discussion}

\subsection{Experiments: confocal microscopy and rheology}

Confocal microscopy was used to determine mean squared displacements (MSDs, equation (1)) under shear for different shear rates $\dot{\gamma}=0.0011,0.0022,0.0038$ and $0.0076 \mathrm{~s}^{-1}$ and a fixed volume fraction $\phi=0.56$. The shear rates can be converted to Weissenberg numbers $P e^{w}=2 \dot{\gamma} R^{2} / D_{\mathrm{L}}^{\mathrm{EQ}}$ by using the long-time diffusion coefficient $D_{\mathrm{L}}^{\mathrm{EQ}}=2.3 \times$ $10^{-13} \mathrm{~cm}^{2} \mathrm{~s}^{-1}$ as determined from the long-time behaviour of the MSD of the quiescent, equilibrated sample at rest (figure 2(a), solid circles), yielding $P e^{w}$ values of 56,113, 194 and 389. For these $P e^{w}$ values, the MSD at rest, the transient MSDs with a waiting time $t_{\mathrm{w}}=0 \mathrm{~s}$ and the MSDs in the steady state of shear have been determined (figure 2(a)). Data, normalized by the particle radius squared, $R^{2}$, are shown as a function of time, $t-t_{\mathrm{W}}$, which is normalized by the structural relaxation time $\tau_{\mathrm{R}}=R^{2} / D_{\mathrm{L}}^{\mathrm{EQ}}=2.56 \times 10^{4} \mathrm{~s}$ (figurc 2). The absolute level of the MSDs of the two highest shear rates have been corrected by subtracting a constant value $\left(0.01 R^{2}\right)$ which accounts for the higher noise level in these confocal images, and hence in the data, when compared to the images for the other shear rates.

At sufficiently long waiting times, $t_{\mathrm{W}}$, when the system has reached the steady state of shear, the MSDs exhibit faster dynamics than at rest, as indicated by the earlier onset of diffusion and the correspondingly larger value of the out-of-cage long-time diffusion coefficient, $D_{\mathrm{L}}^{\mathrm{SS}}$. This is in agreement with previous studies on concentrated colloidal dispersions under flow $[18,24,27]$. With increasing $P e^{w}$, the steady-state dynamics become faster and the long-time diffusion starts earlier, which indicates that the shear rate $\dot{\gamma}$ governs the characteristic timescale of the dynamics. Indced, $D_{\mathrm{L}}^{\mathrm{SS}}$ increases with shear rate $\dot{\gamma}$ or $P e^{\mathrm{w}}$ according to a power law with exponent $v=0.92 ; D_{\mathrm{L}}^{\mathrm{SS}} \sim \dot{\gamma}^{0.92}$ (data not shown). This exponent lies between the values of 1.0 for a fluid dispersion and 0.8 for a glassy sample $[18,24]$.

The transient MSDs for $t_{\mathrm{w}}=0$ initially follow the MSD at rest and then exhibit a super-diffusive regime before, at long times, displaying diffusive behaviour and merging with the steady-state MSDs. With increasing waiting time, the super-diffusion becomes less prominent and the dynamics approach the steady-state curve, while its range remains about constant (figure 3(a)), in agreement with the findings of previous work [27]. Super-diffusion gets more pronounced as $P e^{w}$ increases. This becomes more apparent if the MSDs are plotted against the rescaled strain, $\gamma-\gamma_{w}$ (figure 2(b)), whilst dividing the MSD by the rescaled time, which yields an effective diffusion coefficient, $\mathcal{D} / D_{\mathrm{L}}^{\mathrm{EQ}}=\left\langle\Delta y^{2}(t)\right\rangle \tau_{\mathrm{R}} /(t-$ $\left.t_{\mathrm{w}}\right) 2 R^{2}$. Here the super-diffusive regime is indicated by a positive slope, which occurs after a minimum and before a plateau, the latter reflecting the long-time diffusive regime. The deviation from diffusive behaviour can be quantified by the difference between the value at the minimum, $\mathcal{D}_{\min }$, and the value at the plateau, $\mathcal{D}_{\text {diff }}=D_{\mathrm{L}}^{\mathrm{SS}} / D_{\mathrm{L}}^{\mathrm{EQ}}$ (figures 4 (a) and 2(b), dashed lines). The relative difference between $\mathcal{D}_{\text {min }}$ and $\mathcal{D}_{\text {diff }}$ increases with $P e^{\mathrm{w}}$, reflecting a more pronounced super-diffusion, at least for the three lowest values of $P e^{\mathrm{w}}$. It grows from about $2.7 \%$ at $P e^{\mathrm{w}}=56$ to about $17 \%$ at $P e^{w}=389$

The transient super-diffusive regime is observed over a narrow range of strains only. Its beginning, taken as the strain, $\gamma_{\text {noneq, }}$ at which the transient MSD starts to deviate from the MSD at rest (indicated in figure 2(b) for one value of $\left.P e^{w}\right)$, is almost independent of $P e^{w}-\gamma_{\text {noneq }} \approx 1.5 \%$ (figure 4(a)). Similarly, the transition from super-diffusion to long-time diffusion occurs at $\gamma_{\text {diff }} \approx 6 \%$ (indicated in figure $2(\mathrm{~b})$ for one value of $\left.P e^{\mathrm{w}}\right)$ and $\left\langle\Delta y^{2}\right\rangle / R^{2} \approx 0.1$, which is about the cage size, again almost independent of $P e^{\mathrm{w}}$ (figure 2(a)). The transient super-diffusive regime is thus essentially independent of $P e^{\mathrm{w}}$ occurring at $1.5 \% \lesssim \gamma-\gamma_{\mathrm{w}} \lesssim$ $6 \%$ (figure $5(\mathrm{a})$ ), with the minimum of $\mathcal{D} / D_{\mathrm{L}}^{\mathrm{EQ}}$ at $\gamma_{\min } \approx 2 \%$ and showing a slight increase with $P e^{\mathrm{w}}$ (figure 5(b)). That the 

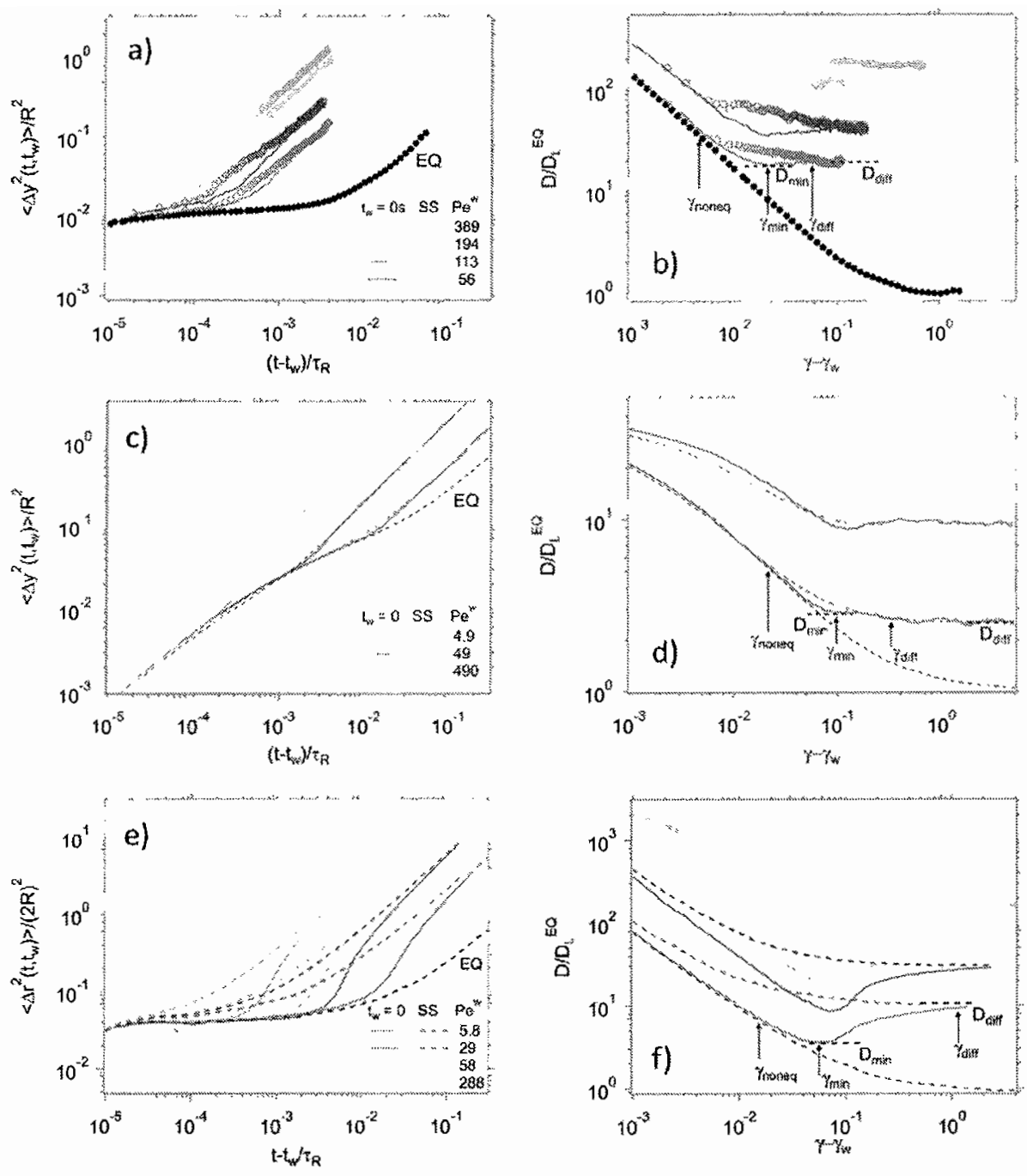

Figure 2. ((a), (c), (e)) Mean squared displacement, in units of the radius squared $R^{2}$, as a function of time $t-t_{\mathrm{w}}$, in units of the structural relaxation time, $\tau_{\mathrm{R}}$. ((b), (d), (f)) The same mean squared displacement normalized by time $t-t_{\mathrm{w}} / \tau_{\mathrm{R}}$, yielding an effective diffusion coefficient $\mathcal{D}$ in units of $D_{\mathrm{L}}^{\mathrm{EQ}}$, as a function of strain $\gamma-\gamma_{\mathrm{w}}$. The data were obtained at rest (denoted as 'EQ'; the corresponding strains in plots (b), (d), and (f) were calculated by multiplying by the lowest shear rate respectively), in the transient regime immediately after application of shear, i.e. waiting time $t_{\mathrm{w}}=0 \mathrm{~s}$ (denoted as ' $t_{\mathrm{w}}=0$ '), and in the steady state of shear (denoted by 'SS') for different values of $P e^{w}$ (as indicated). ((a), (b)) Experiments, $\phi=0.56$, data in the vorticity direction. ((c), (d)) BD simulations, $\phi=0.56$, data in the vorticity direction. ((e), (f)) MD simulations, $T_{\mathrm{c}}=0.14$, data averaged in the vorticity and gradient directions. Arrows indicate the characteristic strains $\gamma_{\text {noneq }}, \gamma_{\text {min }}$ and $\gamma_{\text {diff }}$ whilst the dashed horizontal lines show the values of $\mathcal{D}_{\min }$ and $\mathcal{D}_{\text {diff }}$.

extent of the transient regime is independent of $P e^{\mathrm{w}}$ and $t_{\mathrm{w}}$ suggests that it might be related to some static length scale in the system, possibly the average neighbour separation.

Rheology experiments were performed mainly with smaller particles to increase the signal, which is proportional to the energy density ( $R=267 \mathrm{~nm}$ instead of $770 \mathrm{~nm}$ ). Otherwise the experimental conditions were similar to the confocal measurements, namely $\phi=0.56$ and $P e^{w}=21$, 42,209 and 418, with the last two closely comparable to the two highest $P e^{w}$ in the confocal experiments. We also performed one measurement with the large spheres used for the confocal microscopy experiments at $P e^{\mathrm{W}}=389(\dot{\gamma}=$ $0.0076 \mathrm{~s}^{-1}$ ) to check for consistency of the results. The stress, $\sigma$, was measured as a function of strain, $\gamma$, or time, $t=\gamma / \dot{\gamma}$. Normalized stresses $\left(\sigma / n k_{\mathrm{B}} T\right)$, measured for the large and small spheres at comparable $P e^{w}$ values, show a similar dependence on strain. The absolute values measured for the large spheres are smaller by a factor of about 2 , which can be considered a fair agreement keeping in mind possible differences in volume fraction [46], the different solvents used (i.e. the large spheres could be slightly charged) and a small difference in polydispersity. In addition, the measurement with the large spheres seems to display a smaller stress overshoot in comparison with the data for the small spheres, even though this result could be influenced by the large noise level in the measurements with the large spheres.

In the following we will discuss the results obtained with the small spheres. After a linear regime (figure 6(a)), the 

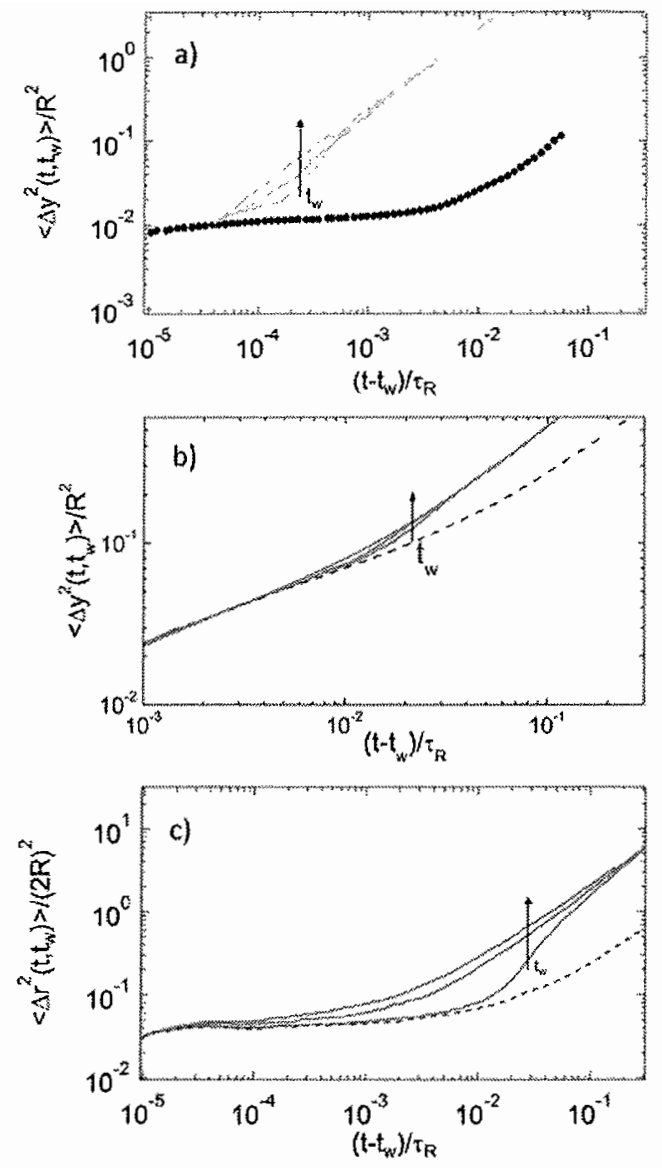

Figure 3. Mean squared displacement, in units of the radius squared, $R^{2}$, as a function of time, $t-t_{\mathrm{w}}$, in units of the structural relaxation time, $\tau_{\mathrm{R}}$, obtained at rest (symbols and dashed lines) and at different waiting times, $t_{\mathrm{w}}$, after application of shear (solid lines). Arrows indicate increasing $t_{\mathrm{w}}$. (a) Experiments, $\phi=0.56$ and $P e^{\mathrm{w}}=194, t_{\mathrm{w}}=0,20$ and $200 \mathrm{~s}$. Data in the vorticity direction. (b) BD simulations, $\phi=0.56$ and $P e^{\mathrm{w}}=4.9, t_{\mathrm{w}}=0,0.5$ and $\mathrm{l}$ (in units of $t_{\mathrm{B}}$ ). Data in the vorticity direction. (c) MD simulations, $T_{\mathrm{c}}=0.14$ and $P e^{\mathrm{w}}=5.8, t_{\mathrm{w}}=0,1342$ and 14763 (simulation units). Data averaged in the vorticity and gradient directions.

stress increases non-linearly and then reaches its maximum $\sigma_{\max }$ at a strain $\gamma_{\max }$, after which it decays to a plateau, $\sigma_{\mathrm{diff}}$. The stress overshoot is quantified by the relative difference between $\sigma_{\max }$ and $\sigma_{\text {diff. }}$. The relative magnitude, quantified by $\left(\sigma_{\max }-\sigma_{\text {diff }}\right) / \sigma_{\text {diff }}$, and strain, $\gamma_{\max }$, of the overshoot increase with $P e^{\mathrm{W}}$ (figures 4 and 5). This may be attributed to the inability of Brownian motion alone to fully relax the distorted structures formed under higher $P e^{w}$ values; hence the ability of the system to store stress, and also the maximum dcformation of the cage before it yields, both increase [26].

Previously, we have shown that the super-diffusion observed in the microscopic dynamics and the stress overshoot in the macroscopic rheology can be linked through negative sections of the generalized shear modulus [27]. The increase in the degree of super-diffusion with increasing $P e^{\mathrm{w}}$ is similar to that of the overshoot, which suggests that the larger stored stress results in a stronger super-diffusive response when the system yields. Super-diffusion and the
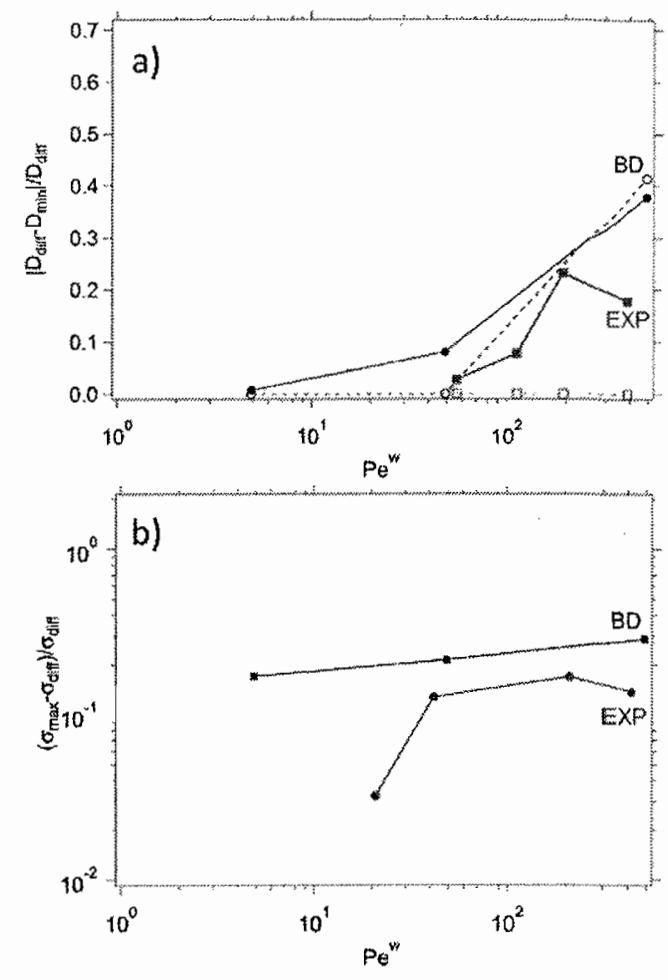

Figure 4. (a) Peclet number $P e^{w}$ dependence of the degree of super-diffusion, quantified by the relative difference between the effective diffusion coefficients at the minimum, $\mathcal{D}_{\min }$, and long-time plateau, $\mathcal{D}_{\text {diff (figures } 2(b) \text {, (d) and (f)). The initial transient }}$ dynamics (filled symbols) and the steady state of shear (open symbols) are presented for the experiments (a), BD simulations $(\bullet)$, and MD simulations ( $₫$ ). (b) Relative magnitude of the stress overshoot, quantified by the difference between the stresses at the peak, $\sigma_{\text {max }}$, and the long-time plateau, $\sigma_{\text {diff }}$ for the experiments, $\mathrm{BD}$ and MD simulations.

overshoot both extend over about half a decade. However, the super-diffusion seems to occur at slightly smaller strains than the stress overshoot, $\gamma_{\min }<\gamma_{\max }$ (figures 2(b), 5(b) and 6(a)). This difference increases with $P e^{\mathrm{w}}$ since the increase in $\gamma_{\max }$ is more strongly dependent on $P e^{w}$ than is the increase in $\gamma_{\min }$ (figure $5(\mathrm{~b})$ ). The observed difference could result from instrumental differences in the synchronization between application of shear and data acquisition in the shear cell and rheometer, or could be the effect of differences between the dynamics of tagged particles, measured in the MSDs, and the collective dynamics related to the macroscopic rheological response.

\subsection{Simulations}

BD simulations of hard spheres are compared to $\mathrm{MD}$ simulations of a binary Yukawa mixture for which Newton's equations of motion are solved. Although colloidal hard spheres are widely used as models for various phenomena in fluids and solids, the extent to which hard spheres exhibit 'universal' features of glass-forming liquids under shear and in particular whether they are a good model for 

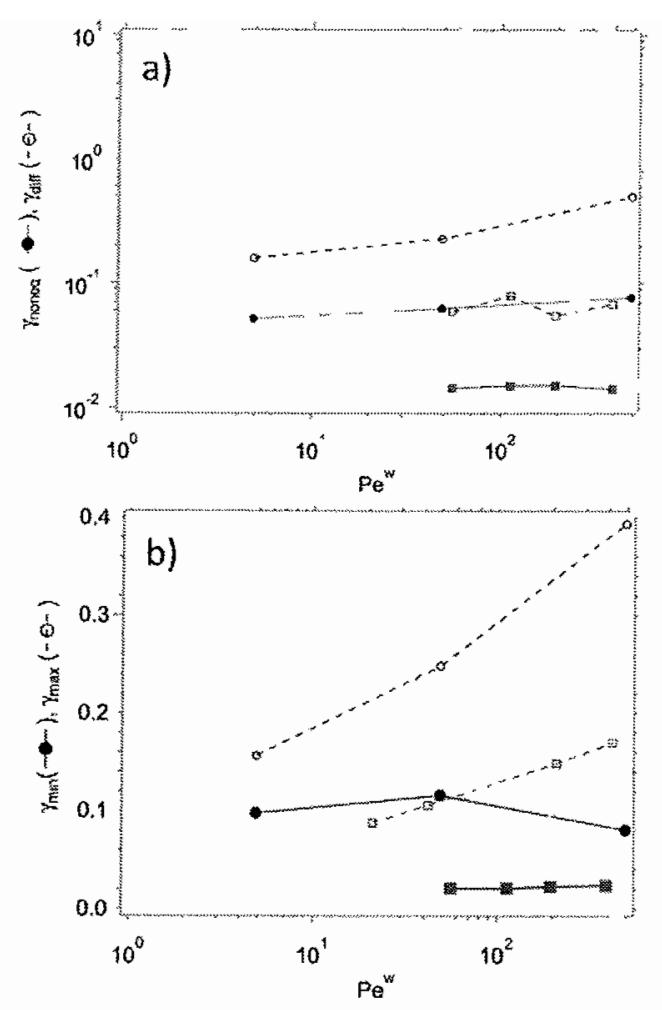

Figure 5. (a) Extent of the transient regime, quantified by the strains at the beginning, $\gamma_{\text {noneq }}$, and end, $\gamma_{\text {diff, }}$, of the transient regime. Symbols as in figure 4. (b) Strain $\gamma_{\min }$ at the minimum of $\mathcal{D}$ for experiments ( $\mathbf{\square}$ ), BD simulations ( $\bullet$ ) and MD simulations ( $\mathbf{\Lambda}$ ), as a function of the Peclet number $P e^{w}$, compared to the strain $\gamma_{\max }$ (open symbols; same colour coding as $\gamma_{\min }$ ) at the peak of the stress overshoot.

sheared molecular glasses is still under debate. Particle-based computer simulation is an ideal tool for inferring similarities between hard spheres and atomistic model systems (such as multicomponent metallic mixtures). Apart from the different interactions between the particles, the microscopic dynamics in atomistic models differ from those in colloidal hard spheres, being Newtonian in the former and Brownian in the latter case.

BD simulations, which apply hard-sphere interactions, and MD simulations based on Yukawa interactions yield qualitatively the same dynamic behaviour, seen here in the MSDs at rest and in the transient and steady state (figures 2(c) and (e)); both agree with the experimental observations. (Note that in the experiments and BD simulations the MSDs are in the vorticity direction, whilst in the MD simulations, the MSDs are averaged over the vorticity and gradient directions.) Both MSDs at rest depart from the plateau at times comparable to the experimental ones. Thus the structural relaxations of the three systems at rest are very similar. In the steady state, both simulations indicate faster dynamics than at rest. The long-time diffusion coefficient $D_{\mathrm{L}}^{\mathrm{SS}}$ increases as $P e^{\mathrm{w}}$ increases, in agreement with the experiments. The transient MSDs show super-diffusion, which becomes less pronounced with increasing waiting time, $t_{\mathrm{w}}$ (figures $3(\mathrm{~b})$ and (c)), in agreement with the experiments and as previously discussed elsewhere [27]. Increasing $P e^{\mathrm{w}}$ enhances super-diffusion in
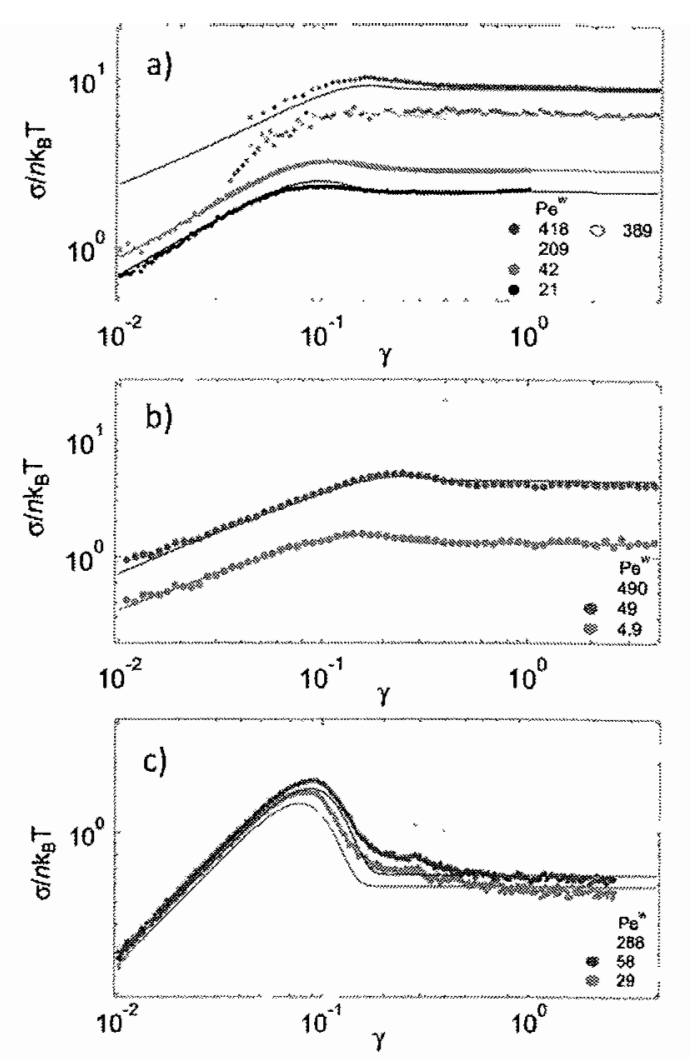

Figure 6. Normalized stress, $\sigma /\left(n k_{\mathrm{B}} T\right)$, as a function of strain, $\gamma$, for different Weissenberg numbers, $P e^{w}$ (as indicated). (a) Experiments, $\phi=0.56$, (filled symbols) spheres of radius $R=267 \mathrm{~nm}$ and (open symbols) spheres of radius $R=770 \mathrm{~nm}$. Lines: MCT fits. (b) BD simulations, $\phi=0.56$ with MCT fits (lines) and (c) MD simulations (symbols), $T=0.14$ with MCT fits (lines).

the $\mathrm{BD}$ simulations (figure 2(d)); the relative difference

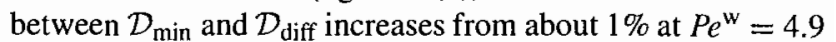
to about $40 \%$ at $P e^{w}=490$. The BD results show a $P e^{w}$ dependence which is qualitatively and almost quantitatively similar to the experiments (figure 4(a)).

However, MD simulations indicate that super-diffusion does not significantly depend on $P e^{\mathrm{w}}$ (figure 2(f)); the relative difference between $\mathcal{D}_{\text {min }}$ and $\mathcal{D}_{\text {diff }}$ remains approximately constant at about 60\% (figure 4(a)), which is larger than in the experiments and BD simulations. This is consistent with a more pronounced stress overshoot in the stress-strain curves found in the MD simulations (figure 6). In addition, neither the stress overshoot nor the super-diffusive behaviour changes significantly with $P e^{\mathrm{w}}$ (figure 4(b)). In the BD simulations, the stress overshoot is less pronounced and similar to that in the experiments. Furthermore, the $\mathrm{BD}$ simulations indicate an increase of the overshoot with $P e^{\mathrm{w}}$ (figures 4(b) and 6(b)), in line with the increasingly pronounced super-diffusion. The more pronounced super-diffusion and stress overshoot in the MD simulations is attributed to the softer Yukawa interactions, compared with the hard-sphere interactions in the BD simulations and experiments. Due to their softness, it is possible to compress particles in their cages and hence to store larger stresses before yielding occurs. A similar 
dependence of the stress overshoot on softness has been found experimentally when comparing hard spheres, microgel particles and star polymers [29]. Note also that in the linear viscoelastic regime at small strains, the stress in the $\mathrm{BD}$ simulations shows a sub-linear dependence on $\gamma$ and some degree of dependence on $P e^{\mathrm{w}}$, whilst stresses in the MD simulations show a linear dependence on $\gamma$ and almost no $P e^{\mathrm{w}}$ dependence. This can be attributed to the different microscopic dynamics: in the BD simulations the short-time Brownian dynamics are reflected in a linear modulus, which depends on $D_{0}$ and is shear rate dependent, and in a finite viscous contribution, which dissipates energy during the elastic deformation (sub-linear increase). Moreover, the absence of diffusion at short times in the MD simulations explains the linear modulus being independent of the shear rate; the Newtonian dynamics do not contribute to the relaxation of structural deformations and stresses. This could also explain the general absence of a $P e^{\mathrm{w}}$ dependence of the super-diffusion and stress overshoot in the MD simulation results.

The super-diffusive regime is limited by $\gamma_{\text {noneq }}$ and $\gamma_{\text {diff }}$. In the BD simulations this regime is shifted to larger strains as compared with the experiments, but shows a similar extent, which increases with increasing $P e^{\mathrm{w}}$ from about $16 \%$ to $43 \%$ (figure $5(\mathrm{a})$ ), with the increase mainly caused by $\gamma_{\text {diff }}$. The difference compared to the experiments might be due to the neglect of the hydrodynamics in the simulations. In the MD simulations, the onset of the transient regime, $\gamma_{\text {noneq }}$, strongly increases with $P e^{w}$, while diffusion is reached at an almost constant value, $\gamma_{\text {diff }} \approx 200 \%$, resulting in an even broader range of strains where transient dynamics and a stress overshoot are found. Furthermore, both simulation techniques find a value of $\gamma_{\min }$ which is larger than in the experiments (figure 5(b)). The ranges of strains where super-diffusion and the stress overshoot occur are comparable in the MD simulations, whilst they do not exactly coincide in the BD simulations and are shifted with respect to each other to an even greater extent in the experiments (figure 5(a)). Since the similar long-time dynamics at rest suggest comparable structural relaxations of the three systems, and since both $\mathrm{BD}$ and $\mathrm{MD}$ simulations neglect hydrodynamic interactions, the different strain ranges might result from the different interactions. In addition, differences in the distance to the glass transition between BD simulations, experiments, and MD simulations could contribute to the discrepancies observed.

In the steady state, super-diffusion disappears completely in the MD simulations and cannot be detected in the experiments. However, in the BD simulations, super-diffusion is observed in the steady state at the highest $P e^{\mathrm{w}}$ studied (figure $2(\mathrm{~d})$ ). The relative difference $\left(\mathcal{D}_{\text {diff }}-\mathcal{D}_{\min }\right) / \mathcal{D}_{\text {diff }} \approx$ $43 \%$ is even larger in the steady state than at $t_{\mathrm{W}}=0$ (figure 4(a)). Super-diffusive behaviour in the steady state has been recently associated with continuous cage breaking and reformation in concentrated hard-sphere dispersions [26].

The BD simulations indicate that, in contrast to the long-time diffusion, the short-time diffusion in the steady state is reduced, compared to that in the transient or quiescent state [26], which becomes more noticeable with increasing $P e^{w}$ (figures 2(c) and (d)). This effect could not be observed in the experiments, due to the limited scanning speed of the confocal microscope, or in the MD simulations, due to the Newtonian dynamics which give rise to a ballistic regime at short times.

\subsection{Mode coupling theory (MCT)}

The experimental and simulation results are compared to mode coupling theory (MCT) using the schematic $F_{12}^{(\dot{\gamma})}$ model (section 2.5). The BD and MD simulations provided rheological data which could be used to set the parameters of the theory (figures 6(b) and (c)). Whilst experimental rheological data are also available, it is not possible to simultaneously fit the stress-strain curves and MSDs as the experiments were performed using small and large particles, respectively, and the fits are quite sensitive to the absolute values of the steady-state stresses, which show some discrepancy at the same nominal volume fraction (figure 6(a)) [46]. Therefore, the rheological data and MSDs from experiments were separately and independently modelled. In the fits to the rheological data, $v_{\sigma}^{*}$ is determined from the linear regions of the stress-strain curves and $\Gamma, \varepsilon$ and the initial value of $\gamma_{c}$ determined from the steady-state stress values of the same curves. Then, $\gamma^{*}$ is chosen as the peak of the stress-strain curves, $\gamma_{\mathrm{c}}$ is fitted to all stress-strain curves and $\gamma^{* *}$ is fitted to each curve. The parameters of the fits are listed in table 1.

For the MSDs, a set of parameters (reported in table 2) is obtained for all data by iteratively fitting theory curves to the experimental data and long-time diffusivities. In doing so, $v_{\sigma}^{*}$ determines the plateau value of the MSD, $\varepsilon$ the plateau width, $\Gamma$ the departure from the plateau at long times, $D_{0}$ the short-time dynamics, $\gamma^{* *}$ and $\gamma_{c}$ the scale for shear induced correlator decay and $\gamma^{*}$ the stress overshoot and the onset of super-diffusive motion.

The theoretical fits reproduce the experimental data at all $P e^{w}$ (figures 6(a) and 7(a)). In particular, they show a stress overshoot and a super-diffusive regime at intermediate times. In agreement with the experiments, stress overshoots and super-diffusion are more pronounced at larger $P e^{\mathrm{w}}$ values. This results from the negative section of the generalized shear modulus, $g(t, \dot{\gamma})$, which becomes more pronounced with increasing $P e^{\mathrm{w}}$ (figure 8, obtained for MSDs, where $m(t, \dot{\gamma}) \propto \alpha g(t, \dot{\gamma})$ from equation (10) is plotted, $\alpha$ being a constant). The transition from super-diffusion to long-time diffusion, however, is not perfectly reproduced. This is attributed to the compressed exponential decay used to describe the time dependence of $v_{\sigma}(t, \dot{\gamma})$ (equation (8)) which describes the stress overshoots very well $[27,59]$, but seems to underestimate the fast process observed in the experimental MSDs. Moreover, fits of the stress-strain curves show some deviations from the data for the largest values of $P e^{w}$. These values of the Weissenberg number are relatively large and therefore the timescales of the short-time and long-time dynamics become close, which means that the limit of applicability of the MCT description is approached. 
Table 1. Parameters of the schematic model used for the fits to the experimental rheology data (figure 6(a)). The first two rows list parameters which are independent of $P e^{w}$ whilst the following rows report the shear-rate-dependent parameters specific to each value of $P e^{w}$.

\begin{tabular}{|c|c|c|c|c|}
\hline \multicolumn{5}{|c|}{ Experimental rheology } \\
\hline \multicolumn{5}{|c|}{ Parameters independent of $P e^{w^{\prime}}$} \\
\hline$v_{\sigma}^{*} /\left[n k_{\mathrm{B}} T\right]=291$ & $\Gamma[s]=19$ & $\varepsilon=-1.4 \times 10^{-2}$ & $\gamma_{c}=0.18$ & $\eta_{\infty} /\left[\left(n k_{\mathrm{B}} T s\right)\right]=6.15$ \\
\hline \multicolumn{5}{|c|}{ Parameters dependent on $P e^{w}$} \\
\hline $\begin{array}{l}P e^{w} \\
\log _{10}(\dot{\gamma}[s] / 6.4) \\
\gamma^{*}\end{array}$ & $\begin{array}{l}21 \\
-3 \\
0.0972\end{array}$ & $\begin{array}{l}42 \\
-3+\log _{10^{2}} 2 \\
0.106\end{array}$ & $\begin{array}{l}209 \\
-2 \\
0.149\end{array}$ & $\begin{array}{l}418 \\
-2+\log _{10} 2 \\
0.171\end{array}$ \\
\hline \multicolumn{5}{|c|}{$\gamma^{* *}=0.983 \gamma^{*}+3.97 \times 10^{-2}$} \\
\hline
\end{tabular}

Table 2. Parameters of the schematic model used for the fits to the experimental MSDs (figure 7(a)). In this case all parameters are independent of $P e^{\mathrm{w}}$.

\begin{tabular}{lllll}
\hline Experimental dynamics (MSDs) & & & \\
\hline$\alpha v_{\sigma}^{*}\left[R^{3} /\left(k_{\mathrm{B}} T\right)\right]=1700 /(6 \pi)$ & $\Gamma[s]=700$ & $\varepsilon=-3 \times 10^{-3}$ & $\gamma_{\mathrm{c}}=0.068$ & $\eta_{\infty}=0$ \\
& & $D_{0}\left[s / R^{2}\right]=0.10$ & $\gamma^{*}=0.022$ & $\gamma^{* *}=0.039$ \\
\hline
\end{tabular}
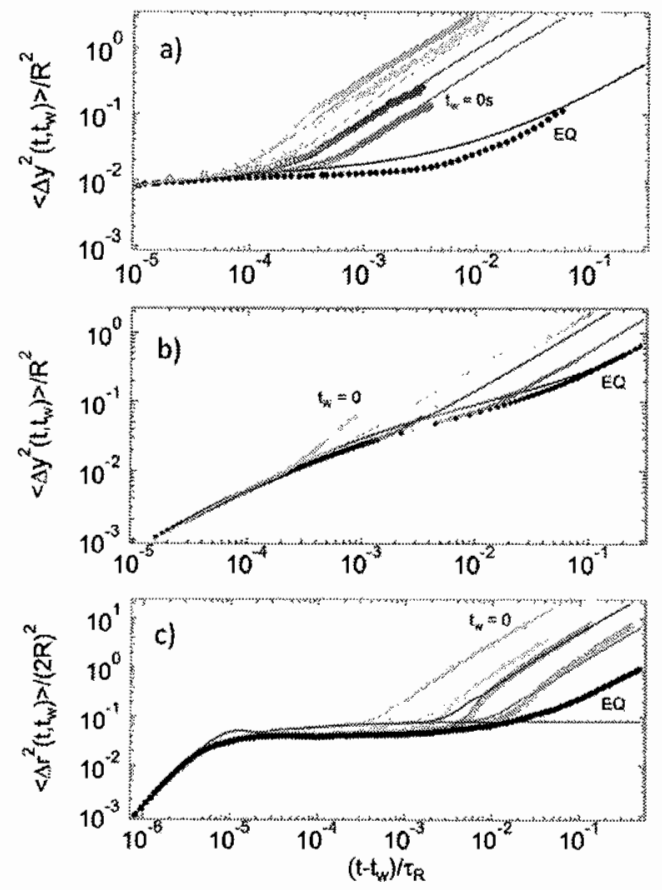

Figure 7. Mean squared displacement, in units of the radius squared, $R^{2}$, as a function of time, $t-t_{\mathrm{w}}$, in units of the structural relaxation time, $\tau_{R}$. The data were obtained at rest (denoted as ' $E Q$ '; full symbols) and in the transient regime immediately after application of shear, i.e. waiting time $t_{\mathrm{w}}=0 \mathrm{~s}\left({ }^{\prime} t_{\mathrm{w}}=0\right.$ '; open symbols) for different values of $P e^{w}$ (the same as in figure 2 ). Mode coupling fits (lines) are compared to (a) experimental results (in the vorticity direction), (b) BD simulation results (in the vorticity direction) and (c) MD simulation results (the average of the vorticity and gradient directions).

For the simulations, the fitting procedure for the stress-strain curves is similar to that for the experiments, except that in the case of MD simulations, values of $v_{\sigma}^{*}$ are obtained by fitting flow curves (not shown) and the

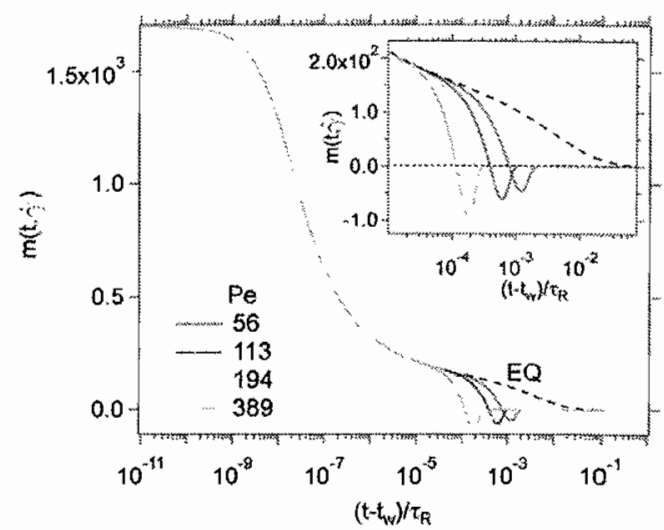

Figure 8. Memory kernel $m(t, \dot{\gamma}) \propto \alpha g(t, \dot{\gamma})$ obtained by fits to the experimental MSDs at equilibrium (EQ) and at different values of $P e^{\mathrm{w}}$ (indicated). Inset: magnified plot of the region of the negative portion of the memory kernel under shear.

same set of parameters used to describe the MSDs. In order to reflect the short-time Newtonian dynamics in the $\mathrm{MD}$ simulations, the microscopic MCT equation (equation (4)) was generalized to generate short-time ballistic motion (as discussed in section 2.5). This allows the MSDs to be successfully reproduced also at short times (figure 7(b)). The parameters of the fits of the BD and MD simulations are reported in table 3 .

The schematic MCT model reproduces the simulated MSDs well and in particular shows super-diffusive behaviour at intermediate times (figures 7(b) and (c)). Only for the BD simulations with $P e^{w}=490$ is the agreement between the MSD and the rheology curve poor. This is due to the fact that the glass transition in the current BD simulations of polydisperse hard spheres is at $\phi=0.6$, i.e. the sample at $\phi=0.56$ is still well in the fluid phase. Therefore, for the high $P e^{w}$ considered, the timescales of the short-time and long-time dynamics are too close to be correctly described by MCT (this is similar to the case for the experimental rheological 
Table 3. Parameters of the schematic model used for the fits to the BD and MD simulations. For BD simulations, the model parameter $D_{0}$ in equation (8) is fitted to the short-lime MSD of figure 7(b).

\begin{tabular}{|c|c|c|c|c|}
\hline \multicolumn{5}{|c|}{ BD simulations } \\
\hline \multicolumn{5}{|c|}{ Parameters independent of $P e^{\mathrm{w}}$} \\
\hline & $\begin{array}{l}v_{\sigma}^{*} /\left[n k_{\mathrm{B}} T\right]=112 \\
\gamma_{c}=0.3\end{array}$ & $\begin{array}{l}\alpha=0.48 \\
\eta_{\infty}=0\end{array}$ & $\Gamma\left[R^{2} / D_{0}\right]=100$ & $\varepsilon=-4.5 \times 10^{-2}$ \\
\hline \multicolumn{5}{|c|}{ Parameters dependent on $\mathrm{Pe}^{\mathrm{w}}$} \\
\hline $\begin{array}{l}P e^{w} \\
\log _{10}\left(\dot{\gamma}\left[R^{2} / D_{0}\right] / 1.29\right) \\
\gamma^{*}\end{array}$ & $\begin{array}{l}4.9 \\
-1 \\
0.16 \quad{ }^{* *}=0.76\end{array}$ & $\begin{array}{l}49 \\
0 \\
0.25 \\
{ }^{*}+1.21 \times\end{array}$ & $\begin{array}{l}490 \\
1 \\
0.36 \\
0^{-1}\end{array}$ & \\
\hline \multicolumn{5}{|c|}{ MD simulations } \\
\hline Parameters independent & $\begin{array}{l}e^{\mathrm{w}} \\
v_{\sigma}^{*} /\left[n k_{\mathrm{B}} T\right]=222 \\
\gamma_{\mathrm{c}}=0.576\end{array}$ & $\begin{array}{l}\alpha=0.18 \\
\eta_{\infty}=0\end{array}$ & $\begin{array}{l}\Omega^{2}\left[\tau^{2}\right]=325 \\
D_{0}\left[\tau / d^{2}\right]=0.41\end{array}$ & $\begin{array}{l}\varepsilon=5 \times 10^{-4} \\
{[\tau]=\sqrt{m d^{2} / \epsilon^{\mathrm{MD}}}}\end{array}$ \\
\hline \multicolumn{5}{|c|}{ Parameters dependent on $P e^{\mathrm{w}}$} \\
\hline $\begin{array}{l}P e^{w} \\
\log _{10} \dot{\gamma}[\tau] / 3 \\
\gamma^{*}\end{array}$ & $\begin{array}{l}5.8 \\
-5+\log _{10} 2 \\
0.078 \quad \gamma^{* *}=1.58\end{array}$ & $\begin{array}{l}29 \\
-4 \\
0.078 \\
*-1.30 \times\end{array}$ & $\begin{array}{l}58 \\
-4+\log _{10} 2 \\
0.088 \\
)^{-2}\end{array}$ & $\begin{array}{l}288 \\
-3 \\
0.11\end{array}$ \\
\hline
\end{tabular}

data at the highest $\left.P e^{\mathrm{w}}\right)$. Moreover, the super-diffusion is significantly less pronounced in the theoretical prediction than in the MD simulations. The discrepancy is larger than between the MCT predictions and the experiments, as expected from the comparison of the $\mathrm{MD}$ simulations and experiments (figure 2, section 3.2) and attributed to the different interactions. Note also that the long-time part of the equilibrium MSD of the MD simulation is not properly modeled by MCT. This is due to the fact that the rheological stress-strain and flow curves show the response of a glass and therefore are described using a positive value of $\varepsilon$ in the MCT model. On the other hand, a glass state (positive $\varepsilon$ ) in MCT is ideal, i.e. it does not show long-time diffusion, contrary to the simulated MSD. We attribute this equilibrium long-time diffusion in the simulated MSD to thermally activated hopping processes, which are not described by MCT. Nevertheless, it is noteworthy that a comparison to the MD and BD rheological and MSD data is possible with identical model parameters, thus quantitatively confirming the relation between the macroscopic rheology and the microscopic dynamics. It should be noted, however, that the difference between the position of the peak of the stress overshoot and the onset of super-diffusion seen in the BD simulation data (and in experiments) is an effect currently not considered by theory.

\section{Conclusions}

Using experiments, $\mathrm{BD}$ and MD simulations as well as MCT, we have investigated concentrated colloidal suspensions, and in particular their macroscopic rheological and microscopic dynamical response to the application of shear with a constant shear rate, or Weissenberg number $P e^{w}$, with a particular focus on the dependence on $P e^{w}$. The transient dynamics exhibit super-diffusion, which occurs between the short-time localized motion and the long-time out-of-cage diffusion. In experiments, BD simulations and MCT, super-diffusion becomes more pronounced with increasing $P e^{\mathrm{w}}$. Upon increasing $P e^{w}$, the timescale introduced by shear (the inverse shear rate) becomes increasingly shorter than the structural relaxation (or $\alpha$-decay) of the system. The internal dynamics of the system thus contribute less and less to the relaxation of structural deformations and stresses. Hence, the cage can store more stress before it yields, as seen in the increase of the stress overshoot in the macroscopic rheological response [26]. An increasingly larger stress is hence released during yielding and super-diffusion is enhanced. MCT explains both trends through the negative section of the generalized shear modulus, which becomes more pronounced with increasing $P e^{\mathrm{w}}$. The degree of super-diffusion is, however, underestimated by MCT, which is due to the chosen compressed exponential time dependence for $v_{\sigma}(t, \dot{\gamma})$ characterizing the strength of fluctuations. Whilst the theory does not capture the fast super-diffusion and, where present, differences in the characteristic strains of the stress overshoot and of the onset of super-diffusion, it reproduces the stress-strain curves very well. In contrast, the MD simulations, which are based on Yukawa interactions, yield only a very weak $P e^{w}$ dependence of both the super-diffusion and the stress overshoot. This could be attributed to the missing short-time diffusion in the Newtonian dynamics and the absence of a short-time dynamics contribution to the relaxation of structural deformations and stresses. Moreover, MD simulations yield stronger stress overshoots compared to experiments and $\mathrm{BD}$ simulations, probably due to the softer interactions involved in the former, in agreement with recent experimental findings [29]. In any case, the super-diffusion and the stress overshoot occur at similar strains. The range of strains is minimal in experiments, larger in BD simulations and largest in MD simulations. This might be caused by the 
neglect of the hydrodynamics (in the simulations) and the softer interactions (in the MD simulations).

\section{Acknowledgments}

This work was funded by the Deutsche Forschungsgemeinschaft (DFG) within the German-Dutch Collaborative Research Centre Sonderforschungsbereich-Transregio 6 (SFB-TR6), Project A6. KJM wishes to thank the Alexander von Humboldt Foundation for financial support through a Research Fellowship. GP and NK acknowledge funding by the Greek General Secretariat for Research and Technology (PENED, -03ED566), the EU Network of Excellence Softcomp and the EU FP7 project 'Nanodirect'. The Crete-Caltech collaboration on $\mathrm{BD}$ simulations was funded by EU ToK 'Cosines'. We finally thank Th Voigtmann for stimulating discussions.

\section{References}

[1] Pusey P N and van Megen W 1986 Phase-behavior of concentrated suspensions of nearly hard colloidal spheres Nature 320 340-2

[2] Weeks E R, Crocker J C, Levitt A C, Schofield A B and Weitz D A 2000 Three-dimensional direct imaging of structural relaxation near the colloidal glass transition Science 287 627-31

[3] Brambilla G, El Masri D, Pierno M, Berthier L, Cipelletti L, Petekidis G and Schofield A B 2009 Probing the equilibrium dynamics of colloidal hard spheres above the mode-coupling glass transition Phys. Rev. Lett. 102085703

[4] Pusey P N and van Megen W 1987 Observation of a glass transition in suspensions of spherical colloidal particles Phys. Rev. Lett. 59 2083-6

[5] van Megen W and Underwood S M 1993 Glass transition in colloidal hard spheres: mode-coupling theory analysis Phys. Rev. Lett. $702766-9$

[6] van Megen W, Mortensen T C, Williams S R and Müller J 1998 Measurement of the self-intermediate scattering function of suspensions of hard spherical particles near the glass transition Phys. Rev. E 58 6073-85

[7] Götze W 1991 Liquids, Freezing and Glass Transition ed J P Hansen, D Levesque and J Zinn-Justin (Amsterdam: North-Holland)

[8] Götze W 2009 Complex Dynamics of Glass-Forming Liquids: A Mode-Coupling Theory (International Series of Monographs on Physics) (Oxford: Oxford University Press)

[9] Kobelev V and Schweizer K S 2005 Strain softening, yielding, and shear thinning in glassy colloidal suspensions Phys. Rev. E 710214201

[10] Mason T G and Weitz D A 1995 Linear viscoelasticity of colloidal hard-sphere suspensions near the glass-transition Phys. Rev. Lett. 75 2770-3

[11] Crassous J J, Siebenburger M, Ballauff M, Drechsler M, Hajnal D, Henrich O and Fuchs M 2008 Shear stresses of colloidal dispersions at the glass transition in equilibrium and in flow J. Chem. Phys. 128204902

[12] Petekidis G, Vlassopoulos D and Pusey P N 2003 Yielding and flow of colloidal glasses Faraday Discuss. 123 287-302

[13] Pham K N, Petekidis G, Vlassopoulos D, Egelhaaf S U, Pusey P N and Poon W C K 2006 Yielding of colloidal glasses Europhys. Lett. 75 624-30

[14] Petekidis G, Vlassopoulos D and Pusey P N 2004 Yielding and flow of sheared colloidal glasses J. Phys.: Condens. Matter 16 S3955-63
[15] Fuchs $M$ and Ballauff M 2005 Flow curves of dense colloidal dispersions: schematic model analysis of the shear-dependent viscosity near the colloidal glass transition J. Chem. Phys. 122094707

[16] Pham K N, Petekidis G, Vlassopoulos D, Egelhaaf S U, Poon W C K and Pusey P N 2008 Yielding behavior of repulsion- and attraction-dominated colloidal glasses J. Rheol. 52 649-76

[17] Varnik F, Bocquet L and Barrat J-L 2004 A study of the static yield stress in a binary Lennard-Jones glass $J$. Chem. Phys. $1202788-80$

[18] Varnik F 2006 Structural relaxation and rheological response of a driven amorphous system J. Chem. Phys, 125164514

[19] Brady J F 1993 The rheological behavior of concentrated colloidal dispersions J. Chem. Phys. 99 567-81

[20] Brader J M, Voigtmann T, Cates M E and Fuchs M 2007 Dense colloidal suspensions under time-dependent shear Phys. Rev. Lett. 98058301

[21] Brader J M, Cates M E and Fuchs M 2008 First-principles constitutive equation for suspension rheology Phys. Rev. Lett. 101138301

[22] Sollich P 1998 Rheological constitutive equation for a model of soft glassy materials Phys. Rev. E 58 738-59

[23] Fielding S M, Sollich P and Cates M E 2000 Aging and rheology in soft materials $J$. Rheol. 44 323-69

[24] Besseling R, Weeks E R, Schofield A B and Poon W C K 2007 Three-dimensional imaging of colloidal glasses under steady shear Phys. Rev. Lett. 99028301

[25] Besseling R, Isa L, Weeks E R and Poon W C K 2009 Quantitative imaging of colloidal flows Adv. Colloid Interface Sci. 146 1-17

[26] Koumakis N, Laurati M, Egelhaaf S U, Brady J F and Petekidis G 2012 Yielding of hard-sphere glasses during start-up shear Phys. Rev. Lett. 108098303

[27] Zausch J, Horbach J, Laurati M, Egelhaaf S U, Brader J M, Voigtmann T and Fuchs M 2008 From equilibrium to steady state: the transient dynamics of colloidal liquids under shear J. Phys.: Condens. Matter 20404210

[28] Derec C, Ducouret G, Ajdari A and Lequex F 2003 Aging and nonlinear rheology in suspensions of polyethylene oxide-protected silica particles Phys. Rev. E 67061403

[29] Koumakis N, Pamvouxoglou A, Poulos A S and Petekidis G 2012 Direct comparison of the rheology of model hard and soft particle glasses Soft Matter 8 4271-84

[30] Osaki K, Inoue T and Isomura T 2000 Stress overshoot of polymer solutions at high rates of shear: polystyrene with bimodal molecular weight distribution J. Polym. Sci. B 38 2043-50

[31] Islam M T and Archer L A 2001 Nonlinear rheology of highly entangled polymer solutions in start-up and steady shear flow J. Polym. Sci. B 39 2275-89

[32] Padding J T, Boek E S and Briels W J 2008 Dynamics and rheology of wormlike micelles emerging from particulate computer simulations J. Chem. Phys. 129074903

[33] Letwimolnun W, Vergnes B, Ausias G and Carreau P J 2007 Stress overshoots of organoclay nanocomposites in transient shear flow J. Non-Newton. Fluid Mech. 141 167-79

[34] Akcora P et al 2009 Anisotropic self-assembly of spherical polymer-grafted nanoparticles Nature Mater: 8 354-9

[35] Mohraz A and Solomon M J 2005 Orientation and rupture of fractal colloidal gels during start-up of steady shear flow J. Rheol. 49 657-81

[36] Carrier V and Petekidis G 2009 Nonlinear rheology of colloidal glasses of soft thermosensitive microgel particles J. Rheol. 53 245-73

[37] Henrich O, Weysser F, Cates M E and Fuchs M 2009 Hard discs under steady shear: comparison of Brownian dynamics simulations and mode coupling theory Phil. Trans. R. Soc. A 367 5033-50 
[38] Zausch J and Horbach J 2009 The build-up and relaxation of stresses in a glass-forming soft-sphere mixture under shear: a computer simulation study Europhys. Lett. 8860001

[39] Krüger M, Weysser F and Fuchs M 2011 Tagged-particle motion in glassy systems under shear: comparison of mode coupling theory and Brownian dynamics simulations Eur. Pllys. J. E 34 I-22

[40] Besseling R, Isa L, Ballesta P, Petekidis G, Cates M E and Poon W C K 2010 Shear banding and flow-concentration coupling in colloidal glasses Phys. Rev. Lett. 105268301

[41.] Ballesta P, Besseling R, Isa L, Petekidis G and Poon W C K 2008 Slip and flow of hard-sphere colloidal glasses Phys. Rev. Lett. 101258301

[42] Yethiraj A and Van Blaaderen A 2003 A colloidal model system with an interaction tunable from hard sphere to sof and dipolar Nature 421 513-7

[43] Jenkins M C and Egelhaaf S U 2008 Confocal microscopy of colloidal particles: towards reliable, optimum coordinates Adv. Colloid Interface Sci. 136 65-92

[44] Pusey P N and Van Megen W 1984 Detection of small polydispersities by photon-correlation spectroscopy J. Chem. Phys. 80 3513-20

[45] Schaertl W and Silescu H 1994 Brownian dynamics of polydisperse colloidal hard-spheres-equilibrium structures and random close packings J. Stat. Phys. 77 1007-25

[46] Poon W C K, Weeks E R and Royall C P 2012 On measuring colloidal volume fractions Soft Matter 8 21-30

[47] Pusey P N 1991 Liquids, Freezing and Glass Transition ed J P Hansen, D Levesque and J Zinn-Justin (Amsterdam: North-Holland)

[48] Petekidis G, Pusey P N, Moussaid A, Egelhaaf S U and Poon W C K 2002 Shear-induced yielding and ordering in concentrated particle suspensions Physica A $306334-42$

[49] Crocker J C and Grier D G 1996 Methods of digital video microscopy for colloidal studies J. Colloid Interface Sci. $179298-310$

[50] Foss D R and Brady J F 1999 Self-diffusion in sheared suspensions by dynamic simulation J. Fluid Mech. $401243-74$
[51] Heyes D M and Melrose J R 1993 Brownian dynamics simulations of model hard-sphere suspensions J. Non-Newton. Fluid Mech. 46 1-28

[52] Foss D R and Brady J F 2000 Brownian dynamics simulation of hard-sphere colloidal dispersions J. Rheol. 44 629-5l

[53] Brady J F 200I Computer simulation of viscous suspensions Chem. Eng. Sci. $562921-6$

[54] Fuchs M and Cates M E 2002 Theory of nonlinear rheology and yielding of dense colloidal suspensions Phys. Rev. Lett. 89248304

[55] Fuchs M and Cates M E 2009 A mode coupling theory for Brownian particles in homogeneous steady shear flow J. Rheol. 53 957-1000

[56] Fuchs M 2010 Nonlinear rheological properties of dense colloidal dispersions close to a glass transition under steady shear Adv. Polym. Sci. 236 55-115

[57] Brader J M, Siebenbürger M, Ballauff M, Reinheimer K, Wilhelm M, Frey S J, Weysser F and Fuchs M 2010 Nonlinear response of dense colloidal suspensions under oscillatory shear: mode-coupling theory and Fourier transform rheology experiments Phys. Rev. E 8206140

[58] Siebenbürger M, Fuchs M, Winter H and Ballauff M 2009 Viscoelasticity and shear flow of concentrated, noncrystallizing colloidal suspensions: comparison with mode-coupling theory J. Rheol. 53 707-26

[59] Amann C, Siebenbürger M, Krüger M, Weysser F, Ballauff M and Fuchs M 2012 Overshoots in stress strain curves: colloidal experiments and schematic mode coupling theory $J$. Rheol. submitted

[60] Fuchs M and Cates M E 2003 Schematic models for dynamic yielding of sheared colloidal glasses Faraday Discuss. $123267-86$

[61] Götze W 1984 Some aspects of phase-transitions described by the self-consistent current relaxation theory Z. Phys. B 56 139-54

[62] Krüger $M$ and Fuchs $M 2010$ Non-equilibrium relation between mobility and diffusivity of interacting Brownian particles under shear Prog. Theor. Phys. Suppl. 184 172-86

[63] Fuchs M, Götzc W and Mayr M R 1998 Asymptotic laws for tagged-particle motion in glassy systems Phys. Rev. E $583384-99$ 